\title{
REPRESENTATION OF FINITE GRAPHS AS DIFFERENCE GRAPHS OF $S$-UNITS, II
}

\author{
K. GYŐRY, L. HAJDU, AND R. TIJDEMAN
}

\begin{abstract}
In part I of the present paper the following problem was investigated. Let $G$ be a finite simple graph, and $S$ be a finite set of primes. We say that $G$ is representable with $S$ if it is possible to attach rational numbers to the vertices of $G$ such that the vertices $v_{1}, v_{2}$ are connected by an edge if and only if the difference of the attached values is an $S$-unit. In part I we gave several results concerning the representability of graphs in the above sense.

In the present paper we extend the results from paper I to the algebraic number field case and make some of them effective. Besides we prove some new theorems: we prove that $G$ is infinitely representable with $S$ if and only if it has a degenerate representation with $S$, and we also deal with the representability with $S$ of the union of two graphs of which at least one is finitely representable with $S$.
\end{abstract}

\section{INTRODUCTION}

In part I of the paper [13] we obtained a variety of theorems on graphs where the vertices have distinct rational values and two vertices are connected by an edge if and only if their values differ by an $S$-unit where $S$ is a given finite set of primes. In this paper we generalize many of these results to the case when the underlying field is not necessarily $\mathbb{Q}$, but any algebraic number field $K$. Moreover, we give effective versions of most of the results. Besides we derive some new results. We study for which sets $S$ a given graph has (infinitely many equivalence classes of) representations with $S$.

All our results in the present paper deal with finite graphs $G$ where the vertices have distinct values from an algebraic number field $K$, and with finite sets $S$ of prime ideals of $K$, such that two vertices of $G$

Date: April 24, 2016.

2010 Mathematics Subject Classification. 05C25, 05C62, 11D61.

Key words and phrases. Arithmetic graphs, cubical graphs, representability, $S$ unit equations.

Research was supported in part by the OTKA grants NK104208 (K.Gy.) and K100339, K115479 (K.Gy. and L.H.). 
are connected by an edge if and only if the difference of their values is an $S$-unit. We call such a graph $G$ a difference graph of $S$-units. In Section 2 we introduce $S$-equivalence of difference graphs, finitely representable and infinitely representable difference graphs, degenerate and non-degenerate representations of difference graphs, and further notation.

In Section 3 we prove that in every number field $K$, for every graph $G$ there is an effectively computable $S$ such that $G$ is representable with $S$. We further deal with graphs with more than one component and with connected graphs which are not doubly connected. In the rest of the paper we restrict ourselves to the case where $G$ is doubly connected. In Section 4 we consider the cases that $G$ is a cycle, a complete bipartite graph or a cubical graph. Section 5 contains the theorem that $G$ is infinitely representable with $S$ if and only if it has a degenerate representation with $S$. In Section 6 two theorems on the union of two graphs are formulated, one for the case that both are finitely representable with $S$, one for the case that only one of them is finitely representable with $S$. Finally, in Section 7 , some effective results are stated for graphs $G$ of which the complement or the attached triangle graph $G^{\triangle}$ satisfy some connectedness condition. In Sections 812 we give the proofs of the statements from Sections 3-7, respectively.

\section{Notation AND TERMinOlOGY}

We introduce notation which will be used throughout the paper.

Let $K$ be an algebraic number field with degree $d$ and discriminant $D_{K}$, and $S$ a finite (possibly empty) set of prime ideals of $K$. We recall that an $\alpha \in K$ is said to be an $S$-integer if in the prime ideal factorization of the ideal $(\alpha)$ generated by $\alpha$ no prime ideal from outside $S$ has negative exponent. The $S$-integers in $K$ form a ring, denoted by $O_{S}$, which is called the ring of $S$-integers. The units $\varepsilon$ of $O_{S}$ (when $\left.\varepsilon, 1 / \varepsilon \in O_{S}\right)$ are called $S$-units. They form a multiplicative group, denoted by $O_{S}^{*}$ and called the group of $S$-units. If in particular $S$ is empty, then $O_{S}$ and $O_{S}^{*}$ are just the ring of integers $O_{K}$ and the unit group $O_{K}^{*}$ of $K$, respectively. Further, in the case $K=\mathbb{Q}$, we denote by $\mathbb{Z}_{S}$ the ring of $S$-integers. In the sequel we suppose that if $S$ is empty, then $K$ is not $\mathbb{Q}$ and not an imaginary quadratic field. Therefore $O_{S}^{*}$ is infinite.

We write

$$
N(S):=\max _{\mathfrak{p} \in S} N(\mathfrak{p}),
$$

where $N(\mathfrak{p})$ stands for the norm of a prime ideal $\mathfrak{p}$. If $a \in K$, then write $h(a)$ for the logarithmic height of $a$, and for $A \subset O_{S}$ with $A=$ 
$\left\{a_{1}, \ldots, a_{n}\right\}$ set

$$
h(A):=\max _{i=1, \ldots, n} h\left(a_{i}\right) .
$$

For any finite ordered subset $A=\left\{\alpha_{1}, \ldots, \alpha_{n}\right\}$ of $O_{S}$, we denote by $\mathcal{G}_{S}(A)$ the graph whose vertices are $\alpha_{1}, \ldots, \alpha_{n}$ and whose edges are the (unordered) pairs $\left\{\alpha_{i}, \alpha_{j}\right\}$ for which

$$
\alpha_{i}-\alpha_{j} \in O_{S}^{*}
$$

cf. Györy [10] where mainly the complements of these graphs were studied. The ordered subsets $A$ and $A^{\prime}$ of $O_{S}$ are called $S$-equivalent if

$$
A^{\prime}=\varepsilon A+\beta
$$

for some $\varepsilon \in O_{S}^{*}$ and $\beta \in O_{S}$. In this case the graphs $\mathcal{G}_{S}(A)$ and $\mathcal{G}_{S}\left(A^{\prime}\right)$ are obviously isomorphic.

Throughout the paper, all graphs we consider are finite and simple. By the order of a graph $G$ we mean the number of its vertices, denoted by $|G|$. We say that a graph $G$ is representable over $K$ with $S$ if there is a subset $A$ of $O_{S}$ such that $\mathcal{G}_{S}(A)$ is isomorphic to $G$. Further, we say that $G$ is effectively representable over $K$ with $S$ if a subset $A$ of $O_{S}$ can be effectively determined such that $\mathcal{G}_{S}(A)$ is isomorphic to $G$. A graph $G$ is called finitely representable over $K$ with $S$ if, up to $S$-equivalence, there are at most finitely many subsets $A$ of $O_{S}$ for which $G$ is isomorphic to $\mathcal{G}_{S}(A)$. Further, $G$ is said to be infinitely representable with $S$ if $G$ is isomorphic to $\mathcal{G}_{S}(A)$ for infinitely many pairwise $S$-nonequivalent $A$. Note that in every representation the vertices have distinct values. In the sequel we omit 'over $K$ ' and 'with $S^{\prime}$ if it is obvious what $K$ and $S$ are.

We note that in all our results on infinite representability with $S$ it suffices that there are more than a certain computable number of equivalence classes which provide representations (cf. Theorem $\mathrm{C}$ and the Remark after its proof in Section 10).

\section{BASIC REPRESENTABILITY THEOREMS}

The first theorem is an effective version of Theorem 2.1 of Part I and, at the same time, a generalization to the number field case.

Theorem 3.1. Let $G$ be a graph with $|G|=n$. Then there exist a finite set $S$ of prime ideals of $K$ and a set $A \subset O_{S}$ with $|A|=n$ such that $G$ is isomorphic to $\mathcal{G}_{S}(A)$, and $N(S) \leq c_{1}\left(n, d, D_{K}\right), h(A) \leq c_{2}\left(n, d, D_{K}\right)$ hold. Here the numbers $c_{i}\left(n, d, D_{K}\right)$ are effectively computable $(i=$ $1,2)$. 
Remark. A simple calculation shows that in case of $K=\mathbb{Q}$ (when $D=d=1)$ bounds of the form $c_{i}(n)=e^{e^{\cdot e^{e}}} \quad$ with $n$ copies of $e(i=$ $1,2)$ apply in the previous theorem. However, since these bounds most probably are very far from the best possible ones, we do not calculate them. An important feature of our proof is that it is constructive: following our argument one can construct $A$ and $S$ with the required property. This is illustrated by an example after the proof of Theorem 3.1 in Section 8.

In what follows, we assume that $K$ is effectively given, i.e. $K=\mathbb{Q}(\vartheta)$ and a minimal polynomial $P \in \mathbb{Q}[x]$ of $\vartheta$ is given. We may assume that $P \in \mathbb{Z}[x]$ and that $\vartheta$ is an algebraic integer. We say that $\alpha \in K$ is effectively given / effectively determinable if in the representation

$$
\alpha=a_{0}+a_{1} \vartheta+\ldots a_{d-1} \vartheta^{d-1}
$$

of $\alpha$ the coefficients $a_{0}, \ldots, a_{d-1} \in \mathbb{Q}$ are given $/$ effectively determinable.

We say that $S$ is effectively given / effectively determinable if the prime ideals in $S$ are effectively given / effectively determinable. This means that a finite set of generators for each prime ideal involved is effectively given / effectively determinable.

Corollary 3.1. Let $G$ be as in Theorem 3.1. Then there is a finite, effectively determinable set $S$ of prime ideals of $K$ such that $G$ is representable with $S$, and some representation of $G$ with $S$ can be, at least in principle, effectively determined.

Theorems 3.2 and 3.3 are effective versions and, at the same time, generalizations to the number field case of the corresponding results of Part I, obtained over $\mathbb{Q}$.

As usual, by a forest graph we mean a graph containing no cycles, i.e. it is a finite, disjoint union of trees.

Theorem 3.2. Let $S$ be any fixed finite set of prime ideals in $K$, and let $G$ be a forest graph with $|G|=n$. Then $G$ is effectively representable with $S$. Further, such a representation $\mathcal{G}_{S}(A)$ can be, at least in principle, effectively constructed such that $h(A) \leq c_{3}\left(n, N(S), d, D_{K}\right)$, where the bound is effectively computable.

In fact, Theorem 3.2 is a simple consequence of the following result.

Theorem 3.3. Let $S$ be any fixed finite set of prime ideals in $K$, and let $A$ be any fixed finite set of $S$-integers, with $|A|=n$.

i) There exist infinitely many $a^{\prime} \in O_{S}$ outside $A$ such that writing $A^{\prime}=A \cup\left\{a^{\prime}\right\}, a^{\prime}$ is an isolated vertex of $\mathcal{G}_{S}\left(A^{\prime}\right)$. 
ii) For every $a \in A$ there exist infinitely many $a^{\prime} \in O_{S}$ such that writing $A^{\prime}=A \cup\left\{a^{\prime}\right\}$, in $\mathcal{G}_{S}\left(A^{\prime}\right)$ the vertex $a^{\prime}$ is connected by an edge with a only.

Further, one can effectively find an element $a^{\prime}$ with either one of the above properties such that $h\left(a^{\prime}\right)<c_{4}\left(n, N(S), d, D_{K}, h(A)\right)$ holds. Here the upper bound is effectively computable.

The next result is a kind of extension of part ii) of Theorem 3.3. We say that a graph is simply connected if it is connected but not doubly connected, and that it is at most simply connected if it is disconnected, or simply connected.

Theorem 3.4. Let $G$ be a graph which is at most simply connected. If $G$ is representable with some $S$, then it is infinitely representable with $S$.

The following result, which is an extension of Theorem 2.4 of Part I to the number field case, shows that the investigations can be reduced to the components of a graph.

Theorem 3.5. Let $S$ be any fixed finite set of prime ideals in $K$, and suppose that every component of a graph $G$ is representable with $S$. Then $G$ is representable with $S$.

\section{Cyclic, Bipartite And CUbical GRAphs}

In view of the results of the previous section, the question of representability of graphs which are not connected, or contain a bridge, is completely settled. So from this point on it is sufficient to consider only graphs not of these types. We say that a graph $G$ having at least one edge is doubly connected, if after deleting any edge of $G$, the graph obtained is connected. If $G$ is not doubly connected, then it is at most simply connected.

In this section we discuss those graphs which are always representable (i.e. representable for all $K$, with all $S$ ). Further, we study certain doubly connected graphs, namely cycles and complete bipartite graphs. The research upon cycles (over $\mathbb{Q}$ ) was initiated by Ruzsa [16]. His intention was, for given $S$, to study the graphs which can be represented with $S$. Besides providing related theorems of various types, Ruzsa also formulated some problems and conjectures. Some of them were solved in [3]. For details see [16, 3].

Let $C_{n}$ denote the cyclic graph of order $n$, and write $K_{m, n}$ for the complete bipartite graph of type $(m, n)$. The next theorem is an extension of Theorem 3.1 of [13] to the number field case. 
Theorem 4.1. i) The graphs $C_{2 n}(n=1,2, \ldots)$ and $K_{2,2}$ are infinitely representable with all $S$.

ii) The graphs $C_{3}, C_{5}$ and $K_{m, n}$ with $m>n>1$ or $m=n \geq 3$ are finitely representable with any $S$.

As one can easily check by examples, it depends on $S$ whether $C_{2 n+1}$ for $n>2$ is infinitely representable.

The following result extends Theorem 3.2 of [13] to the number field case.

Theorem 4.2. If $m>1, n>1$ and

$$
m+n>3 \cdot 2^{16(|S|+d)}
$$

then $K_{m, n}$ is not representable with $S$.

Theorem 4.1 states that there exist graphs which are representable with all $S$, for example $G=K_{2,2}$. In the remaining part of the section we study such graphs.

As we shall see, in this context the so-called cubical graphs play an important role. The $n$-cube $Q_{n}$ is defined in the following way. The vertices of $Q_{n}$ are the $n$-tuples with coordinates 0 and 1, and two vertices are connected by an edge if and only if the vertices differ in exactly one coordinate. It follows that $Q_{n}$ has $2^{n}$ vertices, and $n 2^{n-1}$ edges. An embedding of a graph $G$ into $Q_{n}$ is an injective mapping of the vertices of $G$ into the vertices of $Q_{n}$ such that the edges of $G$ are mapped into the edges of $Q_{n}$. A graph $G$ is called cubical, if it can be embedded in $Q_{n}$ for some $n$. Obviously, cubical graphs are bipartite.

The following theorem is a generalization of Theorem 6.1 of [13] to the number field case.

Theorem 4.3. A graph $G$ is representable with all $K$ and $S$ if and only if $G$ is cubical.

For a survey on related results concerning cubical graphs, we refer to [13].

Remark. It is not true that for fixed $K$, only cubical graphs would be representable with every $S$. Indeed, if $K=\mathbb{Q}(\sqrt{5})$, then since $(1+\sqrt{5}) / 2$ and $(1-\sqrt{5}) / 2$ are both units, a triangle (which is clearly not cubical) is representable with any $S$ as $\{0,1,(1+\sqrt{5}) / 2\}$.

The following result is an extension of Theorem 4.1 from [13]. It plays a crucial role in the proof of Theorem 4.3.

Theorem 4.4. Suppose that a graph $G$ with $|G| \geq 3$ is representable over $\mathbb{Q}$ for some $S$ of the form $S=\{p\}$, where $p$ is a (rational) prime 
larger than twice the number of edges of $G$. Then $G$ is infinitely representable with all $K$ and $S$.

Note that Theorem 4.4 implies that if $G$ is representable with all $K$ and $S$, then $G$ is infinitely representable with all $K$ and $S$.

\section{INFINITE REPRESENTABILITY}

Let $K$ and $S$ be as above. Let $G$ be a graph and $\mathcal{G}_{S}(A)$ a representation of $G$ where $A=\left\{a_{1}, \ldots, a_{n}\right\}$ is the set of vertex values. By a path in $\mathcal{G}_{S}(A)$ we mean a sequence of vertices $a_{i_{1}}, \ldots, a_{i_{m}}$ (repetitions permitted) such that $a_{i_{j}}$ is connected with $a_{i_{j+1}}$ by an edge for $j=1, \ldots, m-1$. We call $a_{i_{1}}$ and $a_{i_{m}}$ the endpoints of the path. We define its path value as $a_{i_{m}}-a_{i_{1}}$. Note that the path value of every closed path (i.e. with $a_{i_{m}}=a_{i_{1}}$ ) is 0 . If $a_{i}$ and $a_{j}$ are connected by an edge, we call the path value from $a_{i}$ to $a_{j}$ the arrow value from $a_{i}$ to $a_{j}$. Hence every edge in $\mathcal{G}_{S}(A)$ generates two arrow values with opposite signs, which we call the arrow values of that edge. Observe further that a path value is the sum of the composing arrow values, $a_{i_{m}}-a_{i_{1}}=\sum_{j=1}^{m-1}\left(a_{i_{j+1}}-a_{i_{j}}\right)$. If $S$ and $A$ are fixed, we write $\mathcal{G}$ for $\mathcal{G}_{S}(A)$. Every representation is meant with respect to $S$.

We shall prove the following properties.

Lemma 5.1. Let $G$ be connected. The equivalence class to which a representation $\mathcal{G}$ of $G$ belongs is determined by its arrow values.

Lemma 5.2. Let $G$ be connected and let values from $K$ be given to all arrows (directed edges) of $G$. Then these arrow values form a representation of $G$ if and only if

1. a path length is 0 if and only if the path is closed and

2. the endpoints of a path are connected by an edge if and only if the path length is in $O_{S}^{*}$.

Remark. Obviously two representations of $G$ are in the same $S$ equivalence class if and only if the quotient of every two corresponding arrow values is the same constant.

Lemma 5.3. Let $\mathcal{G}$ be a representation of a graph $G$. Then there exist only finitely many pairs $(\mathcal{E}, \varepsilon)$ of non-empty proper subsets $\mathcal{E}$ of the set of edges of $\mathcal{G}$ and $S$-units $\varepsilon$ such that multiplying all the arrow values of $\mathcal{E}$ by $\varepsilon$ and leaving all the other arrow values unchanged yields a representation $\mathcal{G}_{\mathcal{E}, \varepsilon}$ of a graph $G_{\mathcal{E}, \varepsilon}$ such that $G$ and $G_{\mathcal{E}, \varepsilon}$ are not isomorphic.

These lemmas are used in the proofs of the following characterizations of graphs with infinitely many representations with $S$. 
Theorem 5.1. Let $G$ be doubly connected and have at least one edge. Then the following statements are equivalent.

a) The graph $G$ is infinitely representable with $S$.

b) There are a representation $\mathcal{G}$ of $G$, a non-empty proper subset $\mathcal{E}$ of the edges of $\mathcal{G}$ and an $S$-unit $\varepsilon_{0} \neq 1$ such that multiplying the arrow values of $\mathcal{E}$ by $\varepsilon_{0}$ and leaving the other arrow values unchanged yields another representation of $G$.

c) There are a representation $\mathcal{G}$ of $G$, a non-empty proper subset $\mathcal{E}$ of the edges of $\mathcal{G}$ and infinitely many $S$-units $\varepsilon$ such that multiplying the arrow values of $\mathcal{E}$ by $\varepsilon$ and leaving the other arrow values unchanged yields another representation of $G$.

We call a representation $\mathcal{G}$ of a graph $G$ degenerate if a non-empty proper subset $\mathcal{E}$ of the edges of $\mathcal{G}$ and infinitely many $S$-units $\varepsilon$ exist such that multiplying the arrow values of $\mathcal{E}$ by $\varepsilon$ and leaving the other arrow values unchanged yields another representation of $G$. Thus Theorem 5.1 has the following consequence.

Corollary 5.1. A doubly connected graph is infinitely representable if and only if it has a degenerate representation.

\section{Finite Representability}

Let in the above notation $K, S$ and $G$ be given. Again representability means representability with $S$. We state some results which can help to establish the finite representability of $G$.

Let $G_{1}, G_{2}$ be induced subgraphs of $G$. We define $G_{1} \cup G_{2}$ as the minimal graph which has all the vertices of $G_{1}$ and $G_{2}$ as vertices and all the edges of $G_{1}$ and $G_{2}$ as edges.

We first treat the case that both $G_{1}$ and $G_{2}$ are finitely representable. Suppose $G=G_{1} \cup G_{2}$ and $G_{1}$ and $G_{2}$ have at most one vertex in common. If either $G_{1}$ or $G_{2}$ is not representable, then $G$ is not representable. If both $G_{1}$ and $G_{2}$ are representable and both have an edge which the other does not have, then $G$ is infinitely representable. Therefore the interesting case is that the intersection of $G_{1}$ and $G_{2}$ consists of at least two vertices. In that case we have the following result.

Theorem 6.1. Suppose $G=G_{1} \cup G_{2}$ and $G_{1}$ and $G_{2}$ are both finitely representable. If $G_{1}$ and $G_{2}$ have at least two vertices in common, then $G$ is finitely representable.

By Theorem 5.1 we know that if $G$ is infinitely representable, then there is a degenerate representation. The following theorem says that 
if $G_{1}$ is finitely representable and both $G_{2}$ and $G_{1} \cup G_{2}$ are infinitely representable, then the degeneracy is entirely in $G_{2} \backslash G_{1}$.

Theorem 6.2. Suppose $G=G_{1} \cup G_{2}$ such that $G_{1}$ is finitely representable and $G_{2}$ is infinitely representable. If $G$ is infinitely representable, then there are a representation $\mathcal{G}$ of $G$, a non-empty subset $\mathcal{E}$ of the edges of $\mathcal{G}$ belonging to the edges of $G \backslash G_{1}$, and infinitely many $S$-units $\varepsilon$ such that multiplying the arrow values of $\mathcal{E}$ by such an $\varepsilon$ and leaving the other arrow values unchanged yields another representation of $G$.

Remark. The remaining case is that both $G_{1}$ and $G_{2}$ are infinitely representable. In that case their union both may be finitely representable and infinitely representable and we do not know a simple criterion to distinguish them. For example, let $K=\mathbb{Q}$ and $S$ consist of odd primes. Consider a cycle $G_{1}$ with 8 vertices, successively $v_{1}, v_{2}, v_{3}, v_{4}, v_{5}, v_{6}, v_{7}, v_{8}$, and a cycle $G_{2}$ with 8 vertices, successively $w_{1}, w_{2}, w_{3}, w_{4}, w_{5}, w_{6}, w_{7}, w_{8}$, such that $G_{1}$ and $G_{2}$ have no edge in common. If $v_{1}=w_{1}, v_{5}=w_{4}$, and $G_{1}$ and $G_{2}$ have no other vertices in common, then the union contains a 7 -cycle $\left(v_{1}, v_{2}, v_{3}, v_{4}, v_{5}=w_{4}, w_{3}, w_{2}\right)$ and therefore there is no representation of $G_{1} \cup G_{2}$ at all. However, if $v_{1}=w_{1}, v_{5}=w_{5}$ and $G_{1}$ and $G_{2}$ have no other vertices in common, then $G_{1} \cup G_{2}$ has two vertices $v_{1}=w_{1}$ and $v_{5}=w_{5}$ which are connected by four disjoint paths of length 4 , and their arrows can be given values $1, p, p^{2}, p^{r}$ and $p, p^{2}, p^{r}, 1$ and $p^{2}, p^{r}, 1, p$ and $p^{r}, 1, p, p^{2}$, respectively, where $r$ is any integer $\geq 3$. Hence $G_{1} \cup G_{2}$ is infinitely representable.

Theorem 6.1 implies two theorems of [13]. The theorems are partial counterparts of Theorem 3.4. For a graph $G$ we denote by $G^{\triangle}$ the graph whose vertices are the edges of $G$, and where two vertices $e_{1}$ and $e_{2}$ of $G^{\triangle}$ are connected by an edge if and only if $G$ contains a triangle having $e_{1}$ and $e_{2}$ as sides. Further, if both $G$ and $G^{\triangle}$ are connected then we say that $G$ is $\triangle$-connected. The $\triangle$-graphs of tree and forest graphs have only isolated vertices. Observe that if both $G$ and $G^{\triangle}$ are connected, then $G$ is doubly connected. For a graph $G$ we denote by $G^{\nabla}$ the graph whose vertices are the triangles of $G$ where two vertices of $G^{\nabla}$ are connected by an edge if and only if the triangles in $G$ have a common side.

Corollary 6.1. Let $G$ be a graph such that $G^{\nabla}$ is non-empty and both $G$ and $G^{\nabla}$ are connected. Then $G$ is finitely representable with every $S$.

The following corollary is a generalization of Theorem 5.1 of Part I to the number field case. 
Corollary 6.2. Let $G$ be a graph such that $G^{\triangle}$ has an edge and both $G$ and $G^{\triangle}$ are connected. Then $G$ is finitely representable with every $S$.

We denote by $\mathcal{H}(G)$ the graph whose vertices are the $\triangle$-connected components of $G$, and two vertices of $\mathcal{H}(G)$ are connected if the corresponding $\triangle$-connected components of $G$ have at least two vertices in common in $G$. This graph $\mathcal{H}(G)$ is called the $\mathcal{H}(G)$-graph of $G$. The next corollary generalizes Theorem 5.2 of Part I to the number field case.

Corollary 6.3. Let $G$ be a graph such that $G$ contains a triangle and both $G$ and $\mathcal{H}(G)$ are connected. Then $G$ is finitely representable with every $S$.

\section{EFFECTIVE RESUlts FOR $\triangle$-CONNECTED GRAPHS}

We give effective versions of Corollaries 6.2 and 6.3.

Theorem 7.1. Let $G$ be a graph of order $n \geq 3$ such that both $G$ and $G^{\triangle}$ are connected. Then, for effectively given $K$ and $S$, all representations of $G$ with $S$ can be effectively determined.

Theorem 7.1 can be generalized in the following way.

Theorem 7.2. Let $G$ be a graph of order $n \geq 3$. Suppose that both $G$ and $\mathcal{H}(G)$ are connected. Then for effectively given $K$ and $S$, all representations of $G$ with $S$ can be effectively determined.

If $G^{\triangle}$ is connected, then $\mathcal{H}(G)$ consists of one vertex and is therefore also connected. Hence Theorem 7.1 is a special case of Theorem 7.2.

The following theorem is a generalization to the number field case and, for $d=1$, an improvement of Theorem 5.3 of Part I. We denote the complement of $G$ by $\bar{G}$.

Theorem 7.3. Let $n \geq 3$ be an integer, and fix $S$. Then for all but at most

$$
\left(n \cdot 5^{114(|S|+d)}\right)^{4(n-1)}
$$

$S$-equivalence classes of ordered $n$-term subsets $A$ from $O_{S}$, one of the following cases holds:

i) $\overline{\mathcal{G}_{S}(A)}$ is connected and at least one of $\mathcal{G}_{S}(A)$ and $\mathcal{G}_{S}(A)^{\triangle}$ is not connected;

ii) $\overline{\mathcal{G}_{S}(A)}$ has exactly two components, $\overline{\mathcal{G}_{1}}$ and $\overline{\mathcal{G}_{2}}$, say, such that $\left|\overline{\mathcal{G}_{1}}\right|=$

1 , and $\mathcal{G}_{2}$ is not connected;

iii) $n=4$ and $\mathcal{G}_{S}(A)=K_{2,2}$. 
As it is pointed out in [10], for each of i), ii), iii), one can choose $S$ such that there are infinitely many $S$-equivalence classes of ordered $n$-term subsets $A$ in $O_{S}$ with the property i), ii), and iii), respectively.

The following corollary is a consequence of Theorem 7.3. This is a generalization and, for $d=1$, an improvement of Theorem 5.4 of Part I to the number field case.

Corollary 7.1. Let $G$ be a graph of order $n \geq 3$ and suppose that $G$ is more than

$$
\left(n \cdot 5^{114(|S|+d)}\right)^{4(n-1)}
$$

times representable for some $S$. Then at least one of $G$ and $G^{\triangle}$ is not connected.

The final theorem is concerned with the situation where no representation is possible.

Theorem 7.4. Let $G$ be a graph of order $n$ such that $\bar{G}$ has either at least three components, or two components of order $\geq 2$. If

$$
n>3 \cdot 2^{16(|S|+d)}
$$

then $G$ is not representable with any $S$.

This is a generalization to the number field case of Theorem 5.5 of Part I.

Question. Does there exist a criterion/algorithm to decide the representability of a graph $G$ for fixed $K$ and $S$ ?

In case of graphs $G$ for which $G$ and $G^{\triangle}$ are connected, Theorem 7.1 gives a positive answer to the algorithmic part of the above question.

\section{Proofs of the Results Stated in SeCtion 3}

In the proofs below we shall work with finite subsets $A$ of $O_{K}$. In every $S$-equivalence class of ordered subsets $A$ from $O_{S}$ there is a set consisting of integers of $K$. Such a subset can be obtained from $A$ by multiplying it by an appropriate element of $O_{S}^{*} \cap O_{K}$. Hence for Theorems 3.1-3.3 it suffices to study the graphs $\mathcal{G}_{S}(A)$ with subsets $A$ having elements from $O_{K}$. In this case, $a, b \in A$ are connected by an edge if and only if $a-b \in O_{S}^{*} \cap O_{K}=O_{K}^{*}$.

Proof of Theorem 3.1. Let $G$ be a fixed graph with $|G|=n$. Let $N_{0}$ be the second smallest norm of prime ideals in $O_{K}$. Note that we certainly have $N_{0} \leq 3^{d}$. Write $n^{\prime}:=\max \left\{n, N_{0}\right\}$ and

$$
S_{0}:=\left\{\mathfrak{p} \text { prime ideal }: N(\mathfrak{p})<n^{\prime}\right\} .
$$


We prove by induction on $k$ that for any graph $G^{\prime}$ with $\left|G^{\prime}\right|=k \leq n$ there exists a finite set $S^{\prime}$ of prime ideals with $S_{0} \subset S^{\prime}$ and a finite set $A^{\prime} \subset O_{K}$ with $\left|A^{\prime}\right|=k$ such that $\mathcal{G}_{S^{\prime}}\left(A^{\prime}\right)$ is isomorphic to $G^{\prime}$. We shall indicate how one can bound the sets $S^{\prime}$ and $A^{\prime}$ in terms of $n, d, D_{K}$.

Let $k=1$. Then $G^{\prime}$ is a graph with one vertex (and without edges). Taking $S^{\prime}=S_{0}$ and $A^{\prime}=\{0\}$, since $N\left(S^{\prime}\right)<n^{\prime}$ and $h\left(A^{\prime}\right)=0$, we are obviously done in this case.

Let now $G^{\prime}$ be a graph such that $\left|G^{\prime}\right|=k$ with $2 \leq k \leq n$. Write $v_{1}, \ldots, v_{k}$ for the vertices of $G^{\prime}$. Let $G^{\prime \prime}$ be the graph obtained from $G^{\prime}$ by omitting the vertex $v_{k}$, together with the corresponding edges. By induction we may assume that there exists a set $S^{\prime \prime}$ of prime ideals containing all elements of $S_{0}$ and a set $A^{\prime \prime}=\left\{a_{1}, \ldots, a_{k-1}\right\}$ of integers in $K$ such that $\mathcal{G}_{S^{\prime \prime}}\left(A^{\prime \prime}\right)$ is isomorphic to $G^{\prime \prime}$, by an isomorphism $\varphi: \mathcal{G}_{S^{\prime \prime}}\left(A^{\prime \prime}\right) \rightarrow G^{\prime \prime}$. Further, here we may also suppose that $N\left(S^{\prime \prime}\right)<c^{\prime \prime}\left(n, d, D_{K}\right)$ and $h\left(A^{\prime \prime}\right)<c^{\prime \prime}\left(n, d, D_{K}\right)$ with some effectively computable constant $c^{\prime \prime}\left(n, d, D_{K}\right)$ depending only on $n, d, D_{K}$. Without loss of generality we may assume that $\varphi\left(a_{i}\right)=v_{i}(i=1, \ldots, k-1)$. Write $T^{\prime \prime}$ for the set of indices of those vertices of $G^{\prime \prime}$ which are not connected with $v_{k}$ by an edge in $G^{\prime}$. Further, put

$$
\mathfrak{D}:=\left\{\mathfrak{d} \text { prime ideal }: \mathfrak{d} \notin S^{\prime \prime}, \mathfrak{d} \mid a-b \text { for some distinct } a, b \in A^{\prime \prime}\right\} .
$$

For later use, observe that for all $\mathfrak{d} \in \mathfrak{D}$ we have $N(\mathfrak{d}) \geq n^{\prime}>k-1$.

If $T^{\prime \prime} \neq \emptyset$, write $T^{\prime \prime}=\left\{t_{1}, \ldots, t_{\ell}\right\}$, and choose distinct prime ideals $\mathfrak{q}_{t_{1}}, \ldots, \mathfrak{q}_{t_{\ell}}$ such that for all $t_{j} \in T^{\prime \prime}$ we have

- $\mathfrak{q}_{t_{j}} \notin S^{\prime \prime}$,

- $\mathfrak{q}_{t_{j}} \notin \mathfrak{D}$.

Observe that having the upper bounds for $N(\mathfrak{q})$ with $\mathfrak{q} \in S^{\prime \prime} \cup \mathfrak{D}$, the prime ideals $\mathfrak{q}_{t_{1}}, \ldots, \mathfrak{q}_{t_{\ell}}$ can be chosen in a way that their norms are bounded in terms of $n, d, D_{K}$. By the above properties, for any distinct $i_{1}, i_{2} \in\{1, \ldots, k-1\}$ we have $a_{i_{1}} \not \equiv a_{i_{2}}\left(\bmod \mathfrak{q}_{t_{j}}\right)$. For each prime ideal $\mathfrak{d} \in \mathfrak{D}$ choose an $x_{\mathfrak{d}} \in O_{K}$ such that for all $i=1, \ldots, k-1$ we have

$$
a_{i} \not \equiv x_{\mathfrak{d}} \quad(\bmod \mathfrak{d}) .
$$

Since $N(\mathfrak{d})>k-1$ for all $\mathfrak{d} \in \mathfrak{D}$, such an $x_{\mathfrak{d}}$ exists. Consider now the following linear system of congruences:

$$
\left\{\begin{array}{lll}
a \equiv x_{\mathfrak{d}} & (\bmod \mathfrak{d}) & (\mathfrak{d} \in \mathfrak{D}) \\
a \equiv a_{t_{j}} & \left(\bmod \mathfrak{q}_{t_{j}}\right) & \left(t_{j} \in T^{\prime \prime}\right) .
\end{array}\right.
$$

By the Chinese Remainder Theorem, this system has infinitely many solutions $a$. Choose $a_{k}$ to be a solution, and let $A^{\prime}=A^{\prime \prime} \cup\left\{a_{k}\right\}$. Here using the information concerning the ideals $\mathfrak{d}$ and $\mathfrak{q}_{t_{1}}, \ldots, \mathfrak{q}_{t_{\ell}}$, we 
may assume that $h\left(a_{k}\right)$ is bounded in terms of $n, d, D_{K}$. Further, put $T^{\prime}=\{1, \ldots, k-1\} \backslash T^{\prime \prime}$ and set

$$
S^{\prime}=S^{\prime \prime} \cup\left\{\mathfrak{p} \text { prime ideal }: \mathfrak{p} \mid a_{k}-a_{i} \text { for some } i \in T^{\prime}\right\} .
$$

We claim that by these choices the graph $\mathcal{G}_{S^{\prime}}\left(A^{\prime}\right)$ is isomorphic to $G^{\prime}$. More precisely, an isomorphism is given by $\varphi^{*}: \mathcal{G}_{S^{\prime}}\left(A^{\prime}\right) \rightarrow G^{\prime}$ with $\varphi^{*}\left(a_{i}\right)=v_{i}(i=1, \ldots, k)$.

Let $i \in\{1, \ldots, k-1\}$. If $i \in T^{\prime}$ then on the one hand, $v_{i}$ and $v_{k}$ are connected by an edge in $G^{\prime}$, and on the other hand, by the definition of $S^{\prime}$ we have that $a_{i}$ and $a_{k}$ are connected in $\mathcal{G}_{S^{\prime}}\left(A^{\prime}\right)$. Assume now that $i \in T^{\prime \prime}$. Then $v_{i}$ and $v_{k}$ are not connected in $G^{\prime}$. However, writing $i=t_{j}$, in view of $\mathfrak{q}_{t_{j}} \notin S^{\prime \prime}$ and $\mathfrak{q}_{t_{j}} \mid a_{k}-a_{i}$, we have that $\mathfrak{q}_{t_{j}} \notin S^{\prime}$. Indeed, otherwise $\mathfrak{q}_{t_{j}} \mid a_{k}-a_{i^{\prime}}$ for some $i^{\prime} \in T^{\prime}$, whence $\mathfrak{q}_{t_{j}} \mid a_{i}-a_{i^{\prime}}$ with distinct $i, i^{\prime} \in\{1, \ldots, k-1\}$. This means that $\mathfrak{q}_{t_{j}} \in S^{\prime \prime} \cup \mathfrak{D}$, which contradicts its definition. Thus $\mathfrak{q}_{t_{j}} \mid a_{k}-a_{i}$ implies that $a_{i}$ and $a_{k}$ are not connected by an edge in $\mathcal{G}_{S^{\prime}}\left(A^{\prime}\right)$.

Finally, we need to check that for any $i, j \in\{1, \ldots, k-1\}, a_{i}$ and $a_{j}$ are connected by an edge in $\mathcal{G}_{S^{\prime}}\left(A^{\prime}\right)$ if and only if they are connected by an edge in $\mathcal{G}_{S^{\prime \prime}}\left(A^{\prime \prime}\right)$. If $a_{i}$ and $a_{j}$ are connected by an edge in $\mathcal{G}_{S^{\prime \prime}}\left(A^{\prime \prime}\right)$ then by $S^{\prime \prime} \subset S^{\prime}$, obviously they are connected by an edge in $\mathcal{G}_{S^{\prime}}\left(A^{\prime}\right)$. Assume now that $a_{i}$ and $a_{j}$ are not connected in $\mathcal{G}_{S^{\prime \prime}}\left(A^{\prime \prime}\right)$. Then there is a prime ideal $\mathfrak{d} \in \mathfrak{D} \backslash S^{\prime \prime}$ dividing $a_{i}-a_{j}$. Observe that, by (3) and $(2), \mathfrak{d} \mid a_{k}-x_{\mathfrak{d}}$ and $\mathfrak{d} \nmid a_{\ell}-x_{\mathfrak{d}}$, whence $\mathfrak{d} \nmid a_{k}-a_{\ell}$ for $\ell=1, \ldots, k-1$. This implies that $\mathfrak{d} \notin S^{\prime}$. Hence $a_{i}$ and $a_{j}$ are not connected by an edge in $\mathcal{G}_{S^{\prime}}\left(A^{\prime}\right)$ either.

As one can see from the construction, $N\left(S^{\prime}\right)$, as well as $h\left(A^{\prime}\right)$ can be bounded effectively in terms of $n, d, D_{K}$. Hence the statement follows.

An example. Here we illustrate (in fact by providing a detailed explanation) the construction given in the above proof through an example. For simplicity, we shall work over $K=\mathbb{Q}$.

Consider first the cyclic graph $C_{4}=\left(v_{1}, v_{2}, v_{3}, v_{4}\right)$ (with points $v_{1}$, $v_{2}, v_{3}, v_{4}$ and edges $\left.\left\{v_{1}, v_{2}\right\},\left\{v_{2}, v_{3}\right\},\left\{v_{3}, v_{4}\right\},\left\{v_{4}, v_{1}\right\}\right)$. As in the above proof, we use $S$ and $A$ as 'variables', to be changed during the algorithm. Recall that the starting set $S=S_{0}$ should contain all primes less than $n$. Since in our case $n=4$, we shall start with the set of primes $\{2,3\}$.

Initialization. We put $S=\{2,3\}$ and $A=\emptyset$.

Step 1 . We can choose any integer $a_{1}$ to represent $v_{1}$; take $a_{1}:=0$, and put $A:=\{0\}$. $S$ is left unchanged. 
Step 2. Since $v_{2}$ is connected with $v_{1}$, here no restriction is needed; we can represent $v_{2}$ by $a_{2}:=1$, and put $A:=\{0,1\} . S$ is left unchanged once again.

Step 3. Since $v_{1}$ and $v_{2}$ are connected, by an edge, we let $D:=\emptyset$. To choose a representative for $v_{3}$, firstly we need to be sure that the representatives of $v_{1}$ and $v_{3}$ are not connected. For this we take an arbitrary prime $q_{1}$ outside $S$ (and also outside $D$; the latter condition is needed to have distinct moduli in the linear congruence system (3)). Here the choice $q_{1}:=5$ is appropriate. Since $D$ is empty, by (3) we may take $a_{3}:=5$, whence $A:=\{0,1,5\}$. Then we need to insert the prime divisors of $a_{3}-a_{2}=4$ into $S$, to make sure that $a_{2}$ and $a_{3}$ (just as $v_{2}$ and $v_{3}$ ) are connected. In the present case this just means that $S$ remains still unchanged.

Step 4. Now since $v_{1}$ and $v_{3}$ are not connected, we have $D:=\{5\}$. Further, $v_{4}$ is not connected with $v_{2}$. So we need to find a prime $q_{1}$, similarly as in Step 3, i.e. such that $q_{1} \notin D \cup S$. So we can take $q_{1}:=7$. Now we need to find $a_{4}$ subject to (3). Since $a_{2}=1$ and we can take $x_{5}:=2$, this now reads as

$$
\begin{cases}a_{4} \equiv 2 & (\bmod 5) \\ a_{4} \equiv 1 & (\bmod 7) .\end{cases}
$$

Hence we can take $a_{4}:=22$, and let $A:=\{0,1,5,22\}$. Finally, we include the prime divisors of $a_{4}-a_{1}=22$ and $a_{4}-a_{3}=17$ to $S$, to get $S:=\{2,3,11,17\}$.

Output. We output $A=\{0,1,5,22\}$ and $S:=\{2,3,11,17\}$. One can easily check that with these choices, $\mathcal{G}_{S}(A)$ is isomorphic to $C_{4}$.

In what follows, we shall need some algorithmic results for the Chinese Remainder Theorem in number fields (see Algorithm 4.2.2, p. 188 in [2]), for finding an $S$-unit of bounded height (see Algorithm 7.4.8, p. 376 in [2]), for listing all prime ideals of bounded norm (see Algorithm 2.3.23, p. 100 in [2]) and for finding $S$-integers of bounded height (which can be reduced e.g. to listing all prime ideals of bounded norm).

Proof of Corollary 3.1. Suppose that $K$ is effectively given in the sense defined in Section 3. By Theorem 3.1 there exist a finite set $S$ of prime ideals of $K$ and a set $A \subset O_{S}$ with $|A|=n$ such that $G$ is isomorphic to $\mathcal{G}_{S}(A)$, and $N(S) \leq c_{1}\left(n, d, D_{K}\right), h(A) \leq c_{2}\left(n, d, D_{K}\right)$, where $c_{1}, c_{2}$ are effectively computable. However, there are only finitely many such finite sets $S$ of prime ideals in $K$, and for each given $S$, there are only finitely many finite subsets $A$ of $O_{S}$ with these properties, and all pairs 
$S, A$ can be effectively determined. Finally, we can select a pair $S, A$ for which $\mathcal{G}_{S}(A)$ is isomorphic to $G$. In this way we find a representation of $G$ with $S$.

Proof of Theorem 3.4. The proof of the statement is similar to the proof of Theorem 4.2 in [13], we only need to use $O_{S}$ in place of $\mathbb{Z}_{S}$.

We shall use the following finiteness result, due to Evertse, [4] at several places.

Theorem A. (Evertse [4]) The $S$-unit equation

$$
\alpha x+\beta y=1
$$

in $x, y \in O_{S}^{*}$ where $\alpha$ and $\beta$ are nonzero elements of $K$ has at most

$$
3 \cdot 7^{d+2|S|}
$$

solutions where $d=[K: \mathbb{Q}]$.

Proof of Theorem 3.3. Write $A=\left\{a_{1}, \ldots, a_{n}\right\}$.

To prove i) choose prime ideals $\mathfrak{q}_{1}, \ldots, \mathfrak{q}_{n}$ in $K$, outside $S$. Note that here we may clearly assume that the norms of these ideals are bounded in terms of $N(S), d$. Consider the system of linear congruences

$$
x \equiv a_{i} \quad\left(\bmod \mathfrak{q}_{i}\right) \quad(i=1, \ldots, n)
$$

in $x \in O_{K}$. By the Chinese Remainder Theorem, this system has infinitely many solutions. Let $a^{\prime} \in O_{K}$ be a solution such that $a^{\prime} \notin A$. Then obviously, $a^{\prime}$ is an isolated vertex of the graph $\mathcal{G}_{S}\left(A^{\prime}\right)$ where $A^{\prime}=$ $A \cup\left\{a^{\prime}\right\}$. Further, it is also clear that one can effectively find such an $a^{\prime}$, with $h\left(a^{\prime}\right)$ bounded by a constant depending only on $n, N(S), d, D_{K}$.

To prove ii), take an arbitrary $a \in A$. Write

$$
D:=\left\{ \pm\left(a_{i}-a_{j}\right): 1 \leq i<j \leq n\right\},
$$

and let $\varepsilon \in O_{S}^{*} \cap O_{K}$ such that $\varepsilon \notin D$, and for any $\eta \in O_{S}^{*} \cap O_{K}$ we also have $\varepsilon+\eta \notin D$. The existence of such an $\varepsilon$ easily follows from Theorem A. Namely, for $d \in D$ the equation $x+y=d$ has only finitely many solutions in $x, y \in O_{S}^{*} \cap O_{K}$, and the number of solutions can be bounded by a constant $c_{0}(s)$ depending only on $s$, see [9]. Avoiding all such elements $u, v$, together with the at most $2\left(\begin{array}{l}n \\ 2\end{array}\right)$ elements of $D$, in fact we can choose $\varepsilon$ in infinitely many ways. We can bound $h(\varepsilon)$ in terms of $n, N(S), d, D_{K}$ as follows. Take an arbitrary $S$-unit $\varepsilon_{0}$ which is not a root of unity, with $h\left(\varepsilon_{0}\right)$ bounded in terms of $N(S), d, D_{K}$. Considering the powers $\varepsilon_{0}^{i}$ with $i=1, \ldots, c_{0}(s)+2\left(\begin{array}{c}n \\ 2\end{array}\right)$, one of them will be an appropriate choice for $\varepsilon$.

Let $a^{\prime}=a+\varepsilon$. Then $a^{\prime} \notin A$, and obviously $a^{\prime}$ and $a$ are connected by an edge in the graph $\mathcal{G}_{S}\left(A^{\prime}\right)$ where $A^{\prime}=A \cup\left\{a^{\prime}\right\}$. Assume that $a^{\prime}$ is 
also connected with some vertex $b \in A$ with $b \neq a$. Then $b-(a+\varepsilon)=$ : $\eta \in O_{S}^{*} \cap O_{K}$. However, this yields $\eta+\varepsilon=b-a$, whence $\eta+\varepsilon \in D$, contradicting the choice of $\varepsilon$. This shows that in the graph $\mathcal{G}_{S}\left(A^{\prime}\right)$ only the vertex $a$ is connected by an edge with the vertex $a^{\prime}$.

Finally, noting that $h\left(a^{\prime}\right) \leq h(a)+h(\varepsilon)$, our claim follows.

Proof of Theorem 3.2. Let $G$ be the disjoint union of the tree graphs $T_{1}, \ldots, T_{k}$. Starting from one vertex $\alpha \in O_{K}$, using part ii) of Theorem 3.3 , we can inductively build up a set $A_{1} \subset O_{K}$ such that $\mathcal{G}_{S}\left(A_{1}\right)$ is isomorphic to $T_{1}$. Then by part i) of Theorem 3.3 we can adjoin an isolated vertex $a^{\prime} \in O_{K}$ to this graph, and then build up a component $A_{2} \subset O_{K}$ (with $a^{\prime} \in A_{2}$ ) such that $\mathcal{G}_{S}\left(A_{2}\right)$ is isomorphic to $T_{2}$. Following this procedure, we can clearly construct a set $A=A_{1} \cup A_{2} \cup \cdots \cup A_{k}$ with the property that $h(A)$ is bounded by a constant $c_{3}\left(n, N(S), d, D_{K}\right)$.

Proof of Theorem 3.5. The proof is similar to the proof of Theorem 2.4 in [13]. We only need to work with $K$ and $O_{S}$ instead of $\mathbb{Q}$ and $\mathbb{Z}_{S}$, respectively.

\section{Proofs of the Results Stated in Section 4}

We shall need the following two theorems. Theorem B will be used in the present section and in Section 12, and Theorem C in Section 10.

Consider first the equation

$$
\alpha_{1} x_{1}+\cdots+\alpha_{n} x_{n}=1 \quad \text { in } x_{1}, \ldots, x_{n} \in O_{S}^{*}
$$

where $\alpha_{1}, \ldots, \alpha_{n}$ are non-zero elements of $K$. A solution $\left(x_{1}, \ldots, x_{n}\right)$ of (7) is called non-degenerate if

$$
\sum_{i \in I} \alpha_{i} x_{i} \neq 0 \text { for each non-empty subset } I \text { of }\{1, \ldots, n\}
$$

and degenerate otherwise. Clearly, if (7) has a degenerate solution then it has infinitely many solutions. Evertse [5] gave the explicit upper bound below for the number, $N_{n}$, of non-degenerate solutions of (7). His bound was generalized, with a slightly weaker bound, by Evertse, Schlickewei and Schmidt [8] and Amoroso and Viada [1] for the case of finitely generated multiplicative subgroups of $K^{*}$ where $K$ is any field of characteristic 0 .

\section{Theorem B.}

$$
N_{n} \leq\left(2^{35} n^{2}\right)^{n^{3}(|S|+d)}
$$

This is Theorem 3 of Evertse [5]. 
Consider now the system of equations

$$
\alpha_{i 1} x_{1}+\cdots+\alpha_{i n} x_{n}=0, \quad i=1, \ldots, m
$$

in $x_{1}, \ldots, x_{n} \in O_{S}^{*}$, where $\alpha_{i j} \in K$ for $i=1, \ldots, m ; j=1, \ldots, n$. This is a generalization of the homogeneous version of equation (7). Two solutions $\left(x_{1}, \ldots, x_{n}\right)$ and $\left(y_{1}, \ldots, y_{n}\right)$ of $(9)$ are called $S$-equivalent if $y_{j}=\varepsilon x_{j} j=1, \ldots, n$ holds for some $\varepsilon \in O_{S}^{*}$. Further, a solution $\left(x_{1}, \ldots, x_{n}\right)$ is called degenerate, if for some proper non-empty subset $I$ of $\{1, \ldots, n\}$

$$
\sum_{j \in I} \alpha_{i j} x_{j}=0 \quad \text { for } i=1, \ldots, m,
$$

and non-degenerate otherwise. If (9) has a degenerate solution, then it has infinitely many $S$-equivalence classes of solutions.

We shall use the following consequence of Theorem 2 of Evertse and Győry [6]. It is a generalization of earlier work of Evertse, van der Poorten and Schlickewei on $S$-unit equations.

Theorem C. The system of equations (9) has only finitely many nondegenerate equivalence classes of solutions.

For more general versions see Laurent [15] and Győry [11].

Proof of Theorem $C$. Let $\left(x_{1}, \ldots, x_{n}\right)$ be a non-degenerate solution of (9). Then at least one of the coefficients $\alpha_{1 n}, \ldots, \alpha_{m n}$ is different from zero. Putting $y_{j}=-x_{j} / x_{n}$ for $j=1, \ldots, n-1,\left(y_{1}, \ldots, y_{n-1},-1\right)$ is $S$ equivalent to the solution $\left(x_{1}, \ldots, x_{n}\right)$. Further, it satisfies the system of equations

$$
\alpha_{i 1} y_{1}+\cdots+\alpha_{i, n-1} y_{n-1}=\alpha_{i n}, \quad i=1, \ldots, m,
$$

such that there is no proper non-empty subset $J$ of $\{1, \ldots, n-1\}$ with $\sum_{j \in J} \alpha_{i j} y_{j}=0$ for $i=1, \ldots, m$. But by Theorem 2 of Evertse and Györy [6], the number of such solutions $\left(y_{1}, \ldots, y_{n-1}\right)$ is finite, which completes the proof.

Remark. Using Theorem B we can derive an explicit upper bound for the number of non-degenerate equivalence classes of solutions of (9).

Proof of Theorem 4.1. i) Let $n$ be a positive integer and $G=C_{2 n}$. Then $C_{2 n}$ is infinitely representable according to the proof by induction of Theorem 3.1 of [16]. Since $G=K_{2,2}$ is isomorphic to $C_{4}$, the statement is also valid for this graph.

ii) Let $G=C_{3}$. Then every representation of $G$ with $S$ corresponds with a normalized equation $x+y=1$ in $x, y \in O_{S}^{*}$. By Theorem A the 
number of solutions of this equation is finite. Therefore $C_{3}$ is finitely representable with $S$.

Let now $G=C_{5}$, and let $\mathcal{G}_{S}(A)$ be a representation of $G$, with $A=\left\{a_{1}, \ldots, a_{5}\right\} \subset K$. Write $u_{1}, \ldots, u_{5}$ for the $S$-units

$$
a_{2}-a_{1}, a_{3}-a_{2}, a_{4}-a_{3}, a_{5}-a_{4}, a_{1}-a_{5} .
$$

Then we have

$$
u_{1}+\cdots+u_{5}=0
$$

Suppose that the left hand side of (10) contains a vanishing subsum. Then there is such a subsum with two terms. Since these terms cannot be consecutive, we may assume that $u_{1}+u_{3}=0$. However, then $a_{4}-a_{1}=a_{3}-a_{2}$ is also an $S$-unit, which implies that $a_{1}$ and $a_{4}$ should also be connected by an edge in $\mathcal{G}_{S}(A)$. Since this is not the case, we conclude that the left hand side of (10) has no vanishing subsums. Now by Theorem $\mathrm{B}$ we get that the number of non-degenerate solutions of equation (10) is finite. Thus $C_{5}$ is finitely representable.

Finally, let $G=K_{m, n}$ with $m>n>1$ or $m=n \geq 3$. Choose two vertices $P, Q$ from the $n$-set of vertices of $G$. Then, after normalization, this yields $m \geq 3$ distinct solutions of the equation $\alpha x+\alpha y=1$ in $x, y \in O_{S}^{*}$, where $\alpha$ is some non-zero element of $K$. By Theorem A this equation has only finitely many solutions. Thus there are only finitely many ways to represent $G$ with $S$.

Proof of Theorem 4.2. Theorem 4.2 is an immediate consequence of the following theorem, since $\overline{K_{m, n}}$ with $m \geq n \geq 2$ has two components each of size $\geq 2$.

Theorem D. (Györy [12]) Let $A$ be an ordered $n$-term subset in $O_{S}$. If

$$
n>3 \cdot 2^{16(|S|+d)}
$$

then $\overline{\mathcal{G}_{S}(A)}$ has at most two components, and one of them is of order at most 1 . Here $d$ is the degree of the underlying number field $K$.

Proof. This is a special case of Theorem 2.3 of [12].

Proof of Theorem 4.4. The proof is similar to the proof of Theorem 4.1 in [13], working with $K$ and $O_{S}$ in place of $\mathbb{Q}$ and $\mathbb{Z}_{S}$, respectively.

Proof of Theorem 4.3. Assume first that $G$ is representable with all $K$ and $S$. Then, by Theorem 6.1 of [13], $G$ is cubical.

Suppose now that $G$ is cubical. Then, by Theorem 6.1 of [13], $G$ is infinitely representable with $K=\mathbb{Q}$ and $S=\{p\}$, where $p$ is as in Theorem 4.4. Hence the statement follows from Theorem 4.4. 


\section{Proofs of the Results stated in Section 5}

Proof of Lemma 5.1. Let $\mathcal{G}$ be a representation of $G$. Fix a vertex $v$ of $G$. Since $G$ is connected, there is a path from $v$ to any other vertex $w$. The vertex value of $w$ is determined by the vertex value of $v$ and the arrow values of a path from $v$ to $w$ (and apparently independent of the chosen path because of the existence of $\mathcal{G}$ ). Hence all the vertex values of any representation of $G$ are fixed by the vertex value of $v$ and the arrow values. Observe that all the generated representations of $G$ are 'shifts' of $\mathcal{G}$ and therefore equivalent by definition.

Proof of Lemma 5.2. Suppose we have a representation $\mathcal{G}$ of $G$. If the length of a path is 0 and its endpoints are not equal, then there are two vertices with the same value, a contradiction. By definition the length of some path in $\mathcal{G}$ is in $O_{S}^{*}$ if and only if the endpoints of the path are connected by an edge in $\mathcal{G}$.

On the other hand, suppose the conditions of the lemma are fulfilled. Fix a vertex $v$ of $G$ and give it a value $a$. For any other vertex $w$ consider some path from $v$ to $w$ and give $w$ the induced value, $b$ say. Since all paths from $v$ to $w$ have the same path value the value $b$ is independent of the chosen path. Thus $b$ is determined by $a$ and the arrow values. Moreover $a \neq b$, and $v$ and $w$ are connected by an edge if and only if $b-a \in O_{S}^{*}$. Thus we have a representation $\mathcal{G}$ of $G$. The equivalence class to which $\mathcal{G}$ belongs is determined by the arrow values.

Proof of Lemma 5.3. Suppose there are infinitely many pairs $(\mathcal{E}, \varepsilon)$ as in the statement of the lemma such that $G_{\mathcal{E}, \varepsilon}$ is not isomorphic to $G$. Since there are only finitely many possibilities to choose $\mathcal{E}$, there exists an $\mathcal{E}$ for which this is true for infinitely many $S$-units $\varepsilon$. Fix this $\mathcal{E}$. Every arrow value of $\mathcal{G}$ is an $S$-unit. After multiplying it by an $S$-unit $\varepsilon$ it becomes again an $S$-unit. Thus every edge of $G$ leads to an edge of $G_{\mathcal{E}, \varepsilon}$. The only reason that $G$ and $G_{\mathcal{E}, \varepsilon}$ are not isomorphic can be that $G_{\mathcal{E}, \varepsilon}$ has an edge where $G$ has no edge.

There are only finitely many edges which can be added to $G$. Therefore we can fix two vertices $v$ and $w$ in $G$ for which there are infinitely many $S$-units $\varepsilon$ such that multiplying the arrow values of $\mathcal{E}$ in $\mathcal{G}$ by $\varepsilon$ and leaving the others unchanged causes an edge in $G_{\mathcal{E}, \varepsilon}$ between $v$ and $w$. Let $r$ and $r_{\varepsilon}$ denote the path values from $v$ to $w$ in $\mathcal{G}$ and $\mathcal{G}_{\mathcal{E}, \varepsilon}$, respectively. Both $r$ and $r_{\varepsilon}$ are independent of the chosen path. Write $r=P+Q$ where $P$ is the contribution of the arrows from $\mathcal{E}$ to a path from $v$ to $w$ in $\mathcal{G}$ and $Q$ is the contribution of the other arrows along that path. Then $r_{\varepsilon}=\varepsilon \cdot P+Q$. Furthermore $P$ and $Q$ are constants (that is, independent of $\varepsilon$ ) and $r_{\varepsilon}$ is an $S$-unit, $\eta$ say. This yields the 
$S$-unit equation $\eta=\varepsilon \cdot P+Q$ with infinitely many solutions in $S$-units $\varepsilon, \eta$. By Theorem A this equation has only finitely many solutions $\varepsilon, \eta$, unless $P Q=0$. We conclude $P Q=0$. If $P=0$, then $r=Q=r_{\varepsilon}$ and therefore there was already an edge in $\mathcal{G}$ between $v$ and $w$. If $Q=0$, than $r=P=\varepsilon^{-1} r_{\varepsilon}$ is also an $S$-unit, and we have the same conclusion.

Proof of Theorem 5.1. Obviously c) implies both a) and b).

a) $\Rightarrow c$ ). Let $G$ be a doubly connected graph with $n$ edges. Suppose there are infinitely many equivalence classes of representations of $G$. Let $\mathcal{G}$ be any representation of $G$. Let $\mathcal{G}$ have arrow values $\pm x_{1}, \ldots, \pm x_{n}$ where the value of each arrow is fixed and nonzero. Since $G$ is doubly connected, every edge of $G$ is part of a cycle and the edges of $G$ are determined by the cycles. Every cycle of $\mathcal{G}$ corresponds to an equation (9) with $\alpha_{i j} \in\{-1,0,1\}$ for all $j, x_{j}$ the corresponding arrow value and $i$ numbering the (finitely many) cycles. Note that, by Lemmas 5.1 and 5.2, there is a bijection between the solutions of (9) and the arrow value sets which generate representations of graphs $G^{\prime}$ which have the same vertices and edges as $G$, but possibly other edges too. By Theorem $\mathrm{C}$ there are only finitely many non-degenerate equivalence classes of solutions. Since we have infinitely many equivalence classes of solutions, there is a degenerate equivalence class of solutions to system (9). This corresponds to a representation $\mathcal{G}$ of $G$, a proper subset $\mathcal{E}$ of the edges of $\mathcal{G}$ and infinitely many $S$-units $\varepsilon$ such that multiplying the arrow values of $\mathcal{G}$ which belong to $\mathcal{E}$ by an $S$-unit $\varepsilon$ and leaving the other arrow values unchanged leaves the sum of the arrow values of every cycle in $\mathcal{G}$ equal to 0 . According to Lemma 5.3 there are only finitely many $S$-units $\varepsilon$ such that the resulting graph $G_{\mathcal{E}, \varepsilon}$ is not isomorphic to $G$. Hence there are infinitely many $S$-units $\varepsilon$ such that multiplying the arrow values of $\mathcal{E}$ by $\varepsilon$ and leaving the other arrow values unchanged yields another representation of $G$.

b) $\Rightarrow$ c). Suppose there are a representation of $\mathcal{G}$ of $G$, a non-empty proper subset $\mathcal{E}$ of the edges of $\mathcal{G}$ and an $S$-unit $\varepsilon_{0} \neq 1$ such that multiplying the arrow values of $\mathcal{E}$ by $\varepsilon_{0}$ and leaving the other arrow values invariant yields another representation of $G$. Consider any closed path in $\mathcal{G}$. Let $P$ be the total contribution of the edges from $\mathcal{E}$ to this closed path and $Q$ the total contribution of the edges not in $\mathcal{E}$. Then both $P+Q=0$ and $\varepsilon_{0} \cdot P+Q=0$ in view of Lemma 5.2. Since $\varepsilon_{0} \neq 1$ we obtain $P=0$. Thus the contribution of the arrow values from $\mathcal{E}$ to any closed path of $\mathcal{G}$ is 0 .

Consider the graph $\mathcal{G}_{\mathcal{E}, \varepsilon}$ which arises by multiplying the arrow values of $\mathcal{E}$ in $\mathcal{G}$ by the $S$-unit $\varepsilon$ and leaving the other arrow values unchanged. 
Since the contribution of the arrow values from $\mathcal{E}$ to the path values of any closed path is 0 , the graph $\mathcal{G}_{\mathcal{E}, \varepsilon}$ is a representation of some graph $G_{\mathcal{E}, \varepsilon}$ in view of Lemma 5.2. By Lemma 5.3 there exist only finitely many $S$-units $\varepsilon$ for which the graph $G_{\mathcal{E}, \varepsilon}$ is not isomorphic with $G$. Thus there are infinitely many $S$-units $\varepsilon$ such that multiplying the arrow values of $\mathcal{E}$ by $\varepsilon$ and leaving the other arrow values invariant yields another representation of $G$. Each such a representation belongs to a different equivalence class of representations of $G$. Thus $G$ is infinitely representable with $S$.

\section{Proofs of the Results Stated in SeCtion 6}

In the proofs we use the following observations. By the definition of equivalence of representations the values of all the vertices of a representation $\mathcal{G}$ with $S$ of some connected graph $G$ are uniquely determined within an equivalence class by the value of one vertex and the value of one arrow. (Conversely, the value of the vertex may be any element of $K$ and the value of the arrow any $S$-unit.) Since vertices have distinct values, within an equivalence class a representation of $G$ is also uniquely determined by the values of two vertices.

Proof of Theorem 6.1. Consider the set of representations of $G=G_{1} \cup$ $G_{2}$ for which two vertices $v_{1}$ and $v_{2}$ in $G_{1} \cap G_{2}$ have fixed distinct values. Then, by the finite representability of $G_{1}$, there are only finitely many representations of $G_{1}$. But for the same reason there exist only finitely many representations of $G_{2}$. Hence there are only finitely many possibilities to give values to the other vertices of $G$. Thus $G$ is finitely representable.

Proof of Theorem 6.2. If $G_{1}$ and $G_{2}$ have no vertices in common, then the statement is trivial. If $G_{1}$ and $G_{2}$ have precisely one vertex in common, then the situation is still simple. Indeed, consider any representation of $G_{1}$ and any degenerate representation of $G_{2}$. Suppose that in these representations of $G_{1}$ and $G_{2}$ the values $a$ and $a^{\prime}$ are attached to the common vertex of $G_{1}$ and $G_{2}$, respectively. Now adding $a^{\prime}-a$ to the values attached to the vertices of $G_{1}$, we get a representation of $G$ of the required form, since $G_{1}$ and $G_{2}$ have no common edge. Thus the infinitely many pairwise non-equivalent representations of $G_{2}$ yield infinitely many non-equivalent representations of $G$ which leave $G_{1}$ unchanged.

So from this point on we may assume that $G_{1}$ and $G_{2}$ have at least two vertices in common. Every representation of $G$ induces a representation of $G_{1}$ and a representation of $G_{2}$. Fix the values of two vertices 
$v, w \in G_{1} \cap G_{2}$ and consider representations of $G$ with these fixed vertex values. Then, by the finite representability of $G_{1}$, there are only finitely many different induced representations of $G_{1}$.

Suppose $G$ is infinitely representable. Then, by Theorem 5.1 , there are a representation $\mathcal{G}$ of $G$, a non-empty proper subset $\mathcal{E}$ of the edges of $\mathcal{G}$ and infinitely many $S$-units $\varepsilon$ such that multiplying the values of the arrows of $\mathcal{E}$ by $\varepsilon$ and leaving the other arrow values of $\mathcal{G}$ unchanged yields another representation of $G$. Since $G_{1}$ has only finitely many representations, there exists a set $E$ of infinitely many $S$-units $\varepsilon$ such that multiplication of the arrow values of $\mathcal{E}$ by $\varepsilon$ and leaving the other arrow values unchanged yields a representation of $G$ such that its restriction to $G_{1}$ is in the equivalence class of some representation $\mathcal{G}_{1}$ of $G_{1}$.

Let $c$ be the difference of the values of $v$ and $w$ in $\mathcal{G}_{1}$ and $c_{\varepsilon}$ the difference after the multiplication of the arrow values of $\mathcal{E}$ by $\varepsilon \in E$ leaving the other arrow values invariant. Then $c \neq 0$ and, since both representations belong to the same equivalence class of $G_{1}, c_{\varepsilon}=\beta_{\varepsilon} c$ where $\beta_{\varepsilon}$ is an $S$-unit. But for these $\varepsilon$ 's we also have along any path in $G_{1}$ from $v$ to $w$ that $c_{\varepsilon}=\varepsilon P+Q$ where $P$ is the sum of the contributions of the arrows in $\mathcal{E}, Q$ the contribution of the arrows not in $\mathcal{E}$, and $P+Q=c$. Thus the equation $\beta_{\varepsilon} c=\varepsilon P+Q$ with constants $c, P, Q$ has infinitely many solutions in $S$-units $\varepsilon, \beta_{\varepsilon}$. By Theorem A this implies $P=0$ or $Q=0$ as $c \neq 0$. If $P=0$, then $c_{\varepsilon}=Q=c$ for infinitely many $\varepsilon$ 's. Then $c_{\varepsilon}=c$ for infinitely many $\varepsilon$ 's. If $Q=0$, then we repeat the above procedure with $\mathcal{E}$ replaced by its complement in $G$ and conclude that $c_{\varepsilon}=c$ for infinitely many $\varepsilon$ 's too. For such $\varepsilon$ 's the vertex values of $v$ and $w$ remain unchanged. But this means that the restriction to $G_{1}$ is the representation $\mathcal{G}_{1}$ itself. Thus $\mathcal{E}$ belongs to $G \backslash G_{1}$.

Remark. In the proof of Theorem $6.2 P=0$ corresponds to the case that no edge of $G_{1}$ belongs to $\mathcal{E}$ and $Q=0$ to the case that all edges of $G_{1}$ belong to $\mathcal{E}$.

Proof of Corollary 6.1. It suffices to notice that by Theorem 4.1 a triangle is finitely representable for any $S$ and that two triangles with a common edge have two vertices in common. Thus Theorem 6.1 can be used inductively.

Proof of Corollary 6.2. We show that $G^{\nabla}$ is connected if and only if $G^{\triangle}$ is connected. Then the statement follows from Corollary 6.1.

For given $G^{\triangle}$ we can construct $G^{\nabla}$ in the following way: replace every triangle of edges by a vertex and connect two vertices by an edge if and only if the corresponding triangles in $G^{\triangle}$ have a vertex in 
common. Conversely, for given $G^{\nabla}$ we can construct $G^{\triangle}$ by replacing every vertex by a triangle such that if the vertices are connected by an edge in $G^{\nabla}$ the corresponding triangles in $G^{\triangle}$ have a common vertex. It is obvious that there is a path in $G^{\triangle}$ between two triangles if and only if there is a path in $G^{\nabla}$ between the corresponding vertices. This proves our claim.

Proof of Corollary 6.3. Apply Theorem 6.1 to the components of $G^{\triangle}$.

\section{Proofs of the Results stated in Section 7}

We shall need some further preliminary results. The following theorem was established in terms of the complements of the graphs $\mathcal{G}_{S}(A)$ which formulation is more useful for certain applications.

Theorem E. (Győry [12]) Let $n \geq 3$ be an integer, and fix $S$. Then for all but at most

$$
\left((n+1)^{4} 2^{16(|S|+d)}\right)^{n-2}
$$

$S$-equivalence classes of ordered n-term subsets $A$ from $O_{S}$, one of the following cases holds:

i) $\overline{\mathcal{G}_{S}(A)}$ is connected and at least one of $\mathcal{G}_{S}(A)$ and $\mathcal{G}_{S}(A)^{\triangle}$ is not connected;

ii) $\overline{\mathcal{G}_{S}(A)}$ has exactly two components, $\overline{\mathcal{G}_{1}}$, and $\overline{\mathcal{G}_{2}}$, say, such that $\left|\overline{\mathcal{G}_{1}}\right|=$ 1 , and $\mathcal{G}_{2}$ is not connected;

iii) $\overline{\mathcal{G}_{S}(A)}$ has exactly two components of orders $\geq 2$.

Proof. This is a consequence of a special case of Theorem 2.2 of [12].

For $n \geq 5$, the following lemma provides an upper bound for the number of cases in Theorem $\mathrm{E}$ iii).

Lemma 12.1. Let $n \geq 5$ be an integer, and let $S$ be fixed. There are at most

$$
\left(n \cdot 5^{453(|S|+d)}\right)^{n-1}
$$

$S$-equivalence classes of ordered $n$-term subsets $A$ in $O_{S}$ for which $\overline{\mathcal{G}_{S}(A)}$ consists of two components, of which one has order $\geq 3$ and the other has order $\geq 2$.

In the proof of Lemma 12.1 we use the following result.

Lemma 12.2. Apart from an $S$-unit factor, there are at most

$$
2^{1053(|S|+d)}
$$


elements $\gamma \in K^{*}$ such that

$$
x+y=\gamma \quad \text { in } x, y \in O_{S}^{*}
$$

has more than two solutions.

For a qualitative version of Lemma 12.2, see Evertse, Győry, Stewart and Tijdeman [7]. For the special case $K=\mathbb{Q}$, see Lemma 10.2 in Part I.

Proof of Lemma 12.2. A combination of the proof of Lemma 10.2 of Part I with $O_{S}$ instead of $\mathbb{Z}_{S}$ and the inequality $N_{3} \leq 5^{444(|S|+d)}$ from Theorem B proves the assertion.

Proof of Lemma 12.1. Following the proof of Lemma 10.1 of Part I with the choice

$$
C_{1}=5^{444(|S|+d)}, C_{2}=3 \cdot 7^{3 d+2|S|}
$$

and working over $O_{S}$ in place of $\mathbb{Z}_{S}$, the assertion follows.

Proof of Theorem 7.3. Combine Theorem E and Lemma 12.1.

Proof of Corollary 7.1. Let $G$ be a graph of order $n \geq 3$ and suppose that $G$ is more than

$$
\left(n \cdot 5^{114(|S|+d)}\right)^{4(n-1)}
$$

times representable for some $S$. This means that $G$ is isomorphic to $\mathcal{G}_{S}(A)$ for as many $S$-equivalence classes of ordered subsets $A$ from $O_{S}$. The assertion immediately follows from Theorem 7.3.

Proof of Theorem 7.4. The theorem immediately follows from Theorem D.

To prove Theorem 7.1, we shall need the following

Theorem F. (Györy [10]) Let $n \geq 3$ be an integer. For given $S$, there are only finitely many $S$-equivalence classes of $n$-term subsets $A$ in $O_{S}$ such that both $\mathcal{G}_{S}(A)$ and $\mathcal{G}_{S}(A)^{\triangle}$ are connected. These classes of $n$-term subsets are effectively determinable.

Proof. This is in fact an immediate consequence of Theorem 1 of Győry [10]. Indeed, if $A$ is a subset of $O_{S}^{n}$ for which $\mathcal{G}_{S}(A)$ and $\mathcal{G}_{S}(A)^{\triangle}$ are connected, then $A$ is $S$-equivalent to a subset $A^{\prime}$ of $O_{S}^{n}$ such that $A^{\prime}$ is of the form $A^{\prime}=\left\{0, \alpha_{2}^{\prime}, \ldots, \alpha_{n}^{\prime}\right\}$ and $\mathcal{G}_{S}\left(A^{\prime}\right)$ and $\mathcal{G}_{S}\left(A^{\prime}\right)^{\triangle}$ are connected. We can now apply Theorem 1 of [10] to the complement of the graph $\mathcal{G}_{S}\left(A^{\prime}\right)$ and the assertion follows. 
Remark. We note that the proof of Theorem 1 of [10] is based on Györy's [9] effective finiteness results on the equation (5). This result gives also an explicit upper bound for the heights of the solutions. This bound has been improved by several people. The best known bound is due to Györy and $\mathrm{Yu}$ [14]. These bounds could be used to obtain quantitative versions of Theorem F.

Proof of Theorem 7.1. Let $G$ be a graph of order $n \geq 3$ such that both $G$ and $G^{\triangle}$ are connected. If $G$ is representable with $S$ and is isomorphic to $\mathcal{G}_{S}(A)$ for some $n$-term $A \subset O_{S}$, then $\mathcal{G}_{S}(A)$ and $\mathcal{G}_{S}(A)^{\triangle}$ must be connected. Now Theorem $\mathrm{F}$ applies and the assertion follows.

Proof of Theorem 7.2. Let $G$ be a graph of order $\geq 3$. Suppose that $G$ is representable with some $S$ and that $G$ and $\mathcal{H}(G)$ are connected. If $G^{\triangle}$ is connected then the assertion follows from Theorem 7.1. Consider the case when $G^{\triangle}$ is not connected. By Theorem 7.1 each $\triangle$-connected component of $G^{\triangle}$ is finitely representable and, for given $S$, each of these representations is effectively determinable. We claim that if two such components are connected in $\mathcal{H}(G)$ then the subgraph of $G$ spanned by these components is also finitely representable, and all representations of this subgraph can be effectively determined.

Indeed, let $\mathcal{G}_{S}(A)$ be a graph isomorphic to $G$ for some subset $A$ of $O_{S}$, and let $\mathcal{G}_{S}(B), \mathcal{G}_{S}\left(B^{\prime}\right)$ be the induced subgraphs of $\mathcal{G}_{S}(A)$, isomorphic to the respective subgraphs of $G$ spanned by the two components under consideration. Then it follows that

$$
b_{1}-b_{2}=\varepsilon \kappa_{b_{1}, b_{2}} \quad \text { and } \quad b_{1}^{\prime}-b_{2}^{\prime}=\eta \kappa_{b_{1}^{\prime}, b_{2}^{\prime}}^{\prime}
$$

for each distinct $b_{1}, b_{2}$ from $B$ and $b_{1}^{\prime}, b_{2}^{\prime}$ from $B^{\prime}$, where $\varepsilon, \eta$ are $S$-units and $\kappa_{b_{1}, b_{2}}, \kappa_{b_{1}^{\prime}, b_{2}^{\prime}}^{\prime}$ can take only finitely many values from $O_{S}$, and these are effectively determinable. But by assumption $B$ and $B^{\prime}$ have two common vertices, which implies that $\eta=\varepsilon \tau$ for some $\tau \in O_{S}$ which may take only finitely many and effectively determinable values. For each $b_{1} \in B$ and $b_{1}^{\prime} \in B^{\prime}$ we have

$$
b_{1}-b_{1}^{\prime}=\left(b_{1}-b_{2}\right)+\left(b_{2}-b_{1}^{\prime}\right)
$$

where $b_{2}$ is a common vertex of $B$ and $B^{\prime}$. This means that up to the factor $\varepsilon, b_{1}-b_{1}^{\prime}$ may take only finitely many and effectively determinable values from $O_{S}$, whence our claim follows.

Finally, we can treat the remaining components by induction, and the assertion follows.

\section{ACKNOWLEDGEMENTS}

We thank the referee for the useful and helpful comments. 


\section{REFERENCES}

[1] F. Amoroso, E. Viada, Small points on subvariaties of a torus, Duke Math. J. 150 (2009), 407-442.

[2] H. Cohen, Advanced Topics in Computational Number Theory (Springer, 2000).

[3] A. Custić, L. Hajdu, D. Kreso, R. Tijdeman, On conjectures and problems of Ruzsa concerning difference graphs of $S$-units, Acta Math. Hungar. 146 (2015), 391-404.

[4] J.-H. Evertse, On equations in $S$-units and the Thue-Mahler equation, Invent. Math. 78 (1984), 561-584.

[5] J.-H. Evertse, The number of solutions of decomposable form equations, Invent. Math. 122 (1995), 559-601.

[6] J.-H. Evertse, K. Györy, On the number of solutions of weighted unit equations, Compositio Math. 66 (1988), 329-354.

[7] J.-H. Evertse, K. Győry, C. L. Stewart, R. Tijdeman, On $S$-unit equations in two unknowns, Invent. Math. 92 (1988), 461-474.

[8] J.-H. Evertse, H.-P. Schlickewei, W.M. Schmidt, Linear equations in variables which lie in a multiplicative group, Annals of Math. 155 (2002), 807-836.

[9] K. Györy, On the number of solutions of linear equations in units of an algebraic number field, Comment. Math. Helv. 54 (1979), 583-600.

[10] K. Győry, On certain graphs composed of algebraic integers of a number field and their applications, I, Publ. Math Debrecen 27 (1980), 229-242.

[11] K. Győry, Some recent applications of $S$-unit equations, Astérisque 209 (1992), 17-38.

[12] K. Györy, On certain arithmetic graphs and their applications to Diophantine problems, Funct. Approx. 39 (2008), 289-314.

[13] K. Győry, L. Hajdu, R. Tijdeman, Representation of finite graphs as difference graphs of S-units, I, J. Combinatorial Theory, Ser A, 127 (2014), 314-335.

[14] K. Györy, K. Yu, Bounds for the solutions of $S$-unit equations and decomposable form equations, Acta Arith. 123 (2006), 9-41.

[15] M. Laurent, Équations diophantiennes exponentielles, Invent. Math. 78 (1984), 299-327.

[16] I.Z. Ruzsa, The difference graph of S-units, Publ. Math. Debrecen 79 (2011), 675-685.

Institute of Mathematics, University of Debrecen

P. O. Box 12, H-4010 Debrecen, Hungary

E-mail address: gyory@science.unideb.hu

Institute of Mathematics, University of Debrecen

P. O. Box 12, H-4010 Debrecen, Hungary

E-mail address: hajdul@science.unideb.hu

Mathematical Institute, Leiden University

Postbus 9512, 2300 RA Leiden, The Netherlands

E-mail address: tijdeman@math.leidenuniv.nl 